\title{
Under Nutrition and Its Use in Prediction of Immunodeficiency in Adults Infected with Human Immunodeficiency Virus in Addis Ababa-Ethiopia
}

\author{
Shimels Hussien ${ }^{1, ~ *, ~ A y a l e w ~ A k l i l u ², ~ K u m l a c h e w ~ A b a t e ~}{ }^{3}$ \\ ${ }^{1}$ World Health Organization, MCH/Nutrition Cluster, Addis Ababa, Ethiopia \\ ${ }^{2}$ Tulane International, PCP/HMIS Unit, Addis Ababa, Ethiopia \\ ${ }^{3}$ United Nations High Commission for Refugees, Public Health Section, Juba, South Sudan \\ Email address: \\ shimelsh@gmail.com (Shimels H.), abatekumlachew@gmail.com (Kumlachew A.), ayru_2010@yahoo.com (Ayalew A.)
}

\section{To cite this article:}

Shimels Hussien, Ayalew Aklilu, Kumlachew Abate. Under Nutrition and Its Use in Prediction of Immunodeficiency in Adults Infected with Human Immunodeficiency Virus in Addis Ababa-Ethiopia. International Journal of Nutrition and Food Sciences.

Vol. 4, No. 4, 2015, pp. 486-492. doi: 10.11648/j.ijnfs.20150404.21

\begin{abstract}
HIV infection and poor nutrition status are interlinked. HIV infected individuals are more vulnerable to under nutrition than the general population. Despite major advances in HIV treatment and survival outcomes, weight loss and wasting remain of significant health concern in people living with HIV. Poor nutritional status in HIV infected individuals is associated with disease progression, increased morbidity and reduced survival even when antiretroviral treatment (ART) is available. HIV and malnutrition have a cumulative effect in weakening the immune system and worsening nutrition status. The objective of this study was to assess the prevalence of under nutrition (wasting) among HIV infected, antiretroviral naïve adults, and the utility of wasting in predicting immunosuppression in Addis Ababa, Ethiopia, 2014. Institution based, cross sectional study was done on a sample of 395 antiretroviral naïve adults attending chronic HIV care programs. Study participants were selected by systematic random sampling. Data was collected using a structured interview questionnaire and anthropometric measurements (weight and height) were taken from all study participants. CD4 cell count was done using standard laboratory method and used as a proxy indicator of immune status. Body mass index (BMI) was correlated with CD4 cell count and receiver operating characteristic curves plotted. Under nutrition was of critical health concern among HIV infected antiretroviral naïve adults. The study showed a $27 \%$ prevalence of wasting with significantly variation by sex. More women were malnourished than men. There was significant association between wasting and immune suppression. However, the sensitivity and specificity of wasting to predict stages of immune suppression was low. HIV infected individuals need special attention for nutrition monitoring, counselling and support.
\end{abstract}

Keywords: Under Nutrition, HIV, Immunity

\section{Introduction}

The twin global epidemics of HIV infection and food insecurity disproportionately affect sub-Saharan Africa, and a significant proportion of patients who require antiretroviral therapy (ART) are malnourished because of a combination of HIV-associated wasting and inadequate nutrient intake. Protein-calorie malnutrition, the most common form of adult malnutrition in the region, is associated with significant morbidity and compounds the immunosuppressive effects of HIV (1). People living with HIV (PLWH) are more vulnerable to malnutrition than the general population. HIV infection and poor nutrition status are interlinked both weakening the immune system. The synergism between HIV and under nutrition leads to poor adherence to treatment and high mortality rate (2).

Individuals with HIV undergo various physiological alterations beginning in the early phase of the infection. Malnutrition was one of the first complications of AIDS to be recognized and one of the most frequent diagnoses in the population infected with HIV. Several studies have demonstrated association between lean body mass depletion/malnutrition and disease progression/patient survival $(3,4)$. The impaired nutritional status of patients with HIV is partially caused by the reduction in calorie intake, the occurrence of opportunistic diseases and the hypercatabolic 
action of the body in an attempt to control viral replication and recompose the immune system (5). The rise in metabolic rate due to HIV infection is $10 \%$ and $20-30 \%$ higher in asymptomatic and symptomatic adults respectively. This increase in energy expenditure will often lead to weight loss. Thus, wasting may occur despite individuals maintaining their usual food intake after acquiring HIV and during all stages of the disease. Besides, through reduction of food intake, impairment of digestion, malabsorption and food insecurity, HIV progressively impairs nutrition status $(6,7,8)$. Among PLWH, malnutrition is associated with incomplete HIV-1 RNA suppression, CD4 cell decline, increased opportunistic infections, hospitalizations and HIV-related mortality. Good nutrition for PLWH has been proven to increase resistance to infection, help PLWH maintain weight, and improve quality of life, drug compliance and efficacy $(9,10)$.

Malnutrition in turn aggravates the effect of HIV infection. It does this by further weakening the immune system and increasing vulnerability to opportunistic infections, thereby accelerating the course of the HIV infection. Impaired immunity reduces the body's resistance to infection. Thus, despite major advances in antiretroviral treatment and survival outcomes, weight loss and wasting remain of significant public health concern among HIV infected groups. Poor nutrition status in PLWH is associated with disease progression, increased morbidity and reduced survival even when antiretroviral therapy is available $(11,12)$. Poor nutrition status at start of antiretroviral therapy has been identified as an independent predictor of mortality irrespective of immune status. Patients who gain weight in the early phase of treatment have improved prognosis (13).

The prevalence of wasting in adults with advanced HIV infection in sub-Saharan Africa is $20-40 \%$. Low body mass index (BMI) was a strong independent predictor of mortality and treatment outcome among people infected with HIV (15, 16). A low BMI at start of ART was an independent predictor of early mortality (i.e. in the first 90 days of therapy) in several studies in sub-Saharan Africa. In Zambia, patients who started ART with a BMI $<16.0 \mathrm{~kg} / \mathrm{m}^{2}$ had 2-fold higher mortality when compared with those above this BMI threshold (17). In Malawi, patients who initiated ART with a BMI $<16 \mathrm{~kg} / \mathrm{m}^{2}$ had a 6-fold increased risk of death at 3 month compared with those with a BMI $\geq 18.5 \mathrm{~kg} / \mathrm{m}^{2}$, and those with a BMI between 16.0 and $16.9 \mathrm{~kg} / \mathrm{m}^{2}$ had a 2-fold increased risk of death (18). In Tanzania, patients with a BMI $<16.0 \mathrm{~kg} / \mathrm{m}^{2}$ at ART initiation had a mortality rate double that of patients with a $\mathrm{BMI} \geq 18.5 \mathrm{~kg} / \mathrm{m}^{2}$ (19).

While malnutrition itself is multifactorial in causation, the most effective treatment for wasting in HIV-infected individuals is antiretroviral therapy. Furthermore, routine weight monitoring in health facilities providing care, treatment and support could lead to an earlier identification of wasting and provision of appropriate interventions including nutrition counseling, therapeutic/supplementary feeding and antiretroviral therapy as indicated $(20,21)$.

WHO clinical stages III and IV have significant effects on the likelihood of malnutrition development. Malnutrition is usually encountered at the advanced phase of HIV infection. Anthropometric measurements like BMI are lower in symptomatic HIV patients as classified by WHO HIV disease stages, and PLWH with more than $350 \mathrm{CD} 4$ cells/mm3 were more well-nourished than those with less than or equal to 350 CD4 cells/mm3 (22).

Surrogate measures such as the CD4 count and viral load have remained the major markers of disease progression and well-being of PLWH. In many countries of sub-Saharan Africa, CD4 count and viral load measurements are not widely available or consistently used, and instead the WHO clinical staging system is used to determine ART eligibility (23). Where CD4 count is not immediately available, the WHO clinical staging system is used for initial assessment of ART eligibility and monitoring disease progression $(24,25)$. One of the stage-defining conditions to determine eligibility and initiation of ART is wasting (26).

Taking this complex nature of the problem in to account, this study was carried out to assess the prevalence of wasting in PLWH and the extent at which immune suppression could be predicted by wasting. There is limited data on prevalence of under nutrition (wasting) among HIV-infected adults in Ethiopia though it is known that under nutrition is one of the commonest manifestations of HIV in this age group. High rates of malnutrition in Ethiopia pose significant challenges to HIV care and treatment programs (27). We investigated the prevalence of wasting among antiretroviral naïve HIV infected adults. Besides, the study evaluated whether wasting can predict immune deficiency level and serve as surrogate marker of HIV disease severity in peripheral and primary health care settings where facilities for sophisticated laboratory monitoring of viral load and CD4 count are not available.

\section{Method}

\subsection{Study Area and Period}

The study was conducted from July to August, 2014 in Addis Ababa city, Ethiopia. Zewditu hospital was one of the centers which provide chronic HIV care and treatment. The hospital was pioneer with largest antiretroviral treatment and referral center in Ethiopia where over 18,000 HIV infected people had been attending chronic care services (28). The treatment center has been following a cohort of HIV-infected people since 2005 providing comprehensive HIV prevention, care and treatment services. The services included HIV counselling and testing (HCT), prevention of mother to child transmission (PMTCT), antiretroviral therapy (ART), palliative care (PC); and nutrition assessment, counselling and support (NACS). The center also provided a facility based nutrition program called Food by Prescription (FBP) which enabled malnourished HIV infected individuals to receive therapeutic and/or supplementary feeding products.

\subsection{Study Design, Sample and Sampling}

Institution based cross sectional study was conducted 
among a sample of 395 adults. The study participants were HIV infected adults who did not start taking antiretroviral treatment. However, they were receiving other packages of HIV care and support services like co-trimoxazole preventive therapy (CPT), isoniazid preventive therapy (IPT), palliative care and other clinical services as indicated for their clinical conditions.

The assumptions used in calculating the sample size were $95 \%$ confidence level, $5 \%$ margin of error, $10 \%$ non-response rate, and target population of 18,000. Due to the lack of data on prevalence of wasting among HIV infected adults, a default value of $50 \%$ prevalence was used in estimation of the sample size which provided the maximum possible size. The study participants were selected by systematic random sampling during chronic care visits at the treatment center. Every $20^{\text {th }}$ individuals attending pre ART follow up visits were included in the study.

\subsection{Data Collection}

A structured questionnaire was used to interview study participants. The questionnaire incorporated bio data, sociodemography and clinical information. The recent CD4 cell measurement was taken from pre-ART register of the hospital and used as proxy indicator to judge the immunity status of study participants. A normal CD4 cell count ranges from 500 to 1,500 cells per cubic millimeters of blood. Besides, anthropometric measurements were taken on height and weight following standard procedures. Weight was measured by electronic digital weight scale with light clothing and read to the nearest $0.1 \mathrm{~kg}$. Calibration was done routinely before every measurement using an object of known weight. Height was measured using standard standiometer for adults. All the subjects stood against the wall without foot wear and with heels together and their heads positioned so that the line of vision was perpendicular to the body. Height was recorded to the nearest $0.5 \mathrm{~cm}$. Then, the weight and height of each individual were translated into body mass index (BMI), the standard index for assessing nutrition status in adults.

\subsection{Data Analysis}

Data entry into computer was done using CDC Epi-info ${ }^{\circledR}$ version 7. Analysis was done using the program Statistical Package for the Social Sciences (SPSS $®$ ) version 22.The data was used to determine the levels of nutrition status, immunity, and relation between nutrition status and immunity. The WHO BMI cutoff points, $<16 \mathrm{Kg} / \mathrm{m}^{2}$ severe wasting, 16 to 16.99 $\mathrm{Kg} / \mathrm{m}^{2}$ moderate wasting, 17 to $18.49 \mathrm{Kg} / \mathrm{m}^{2}$ mild wasting, 18.5 to $24.99 \mathrm{Kg} / \mathrm{m}^{2}$ normal, 25 to $29.99 \mathrm{Kg} / \mathrm{m}^{2}$ overweight and $\geq 30 \mathrm{Kg} / \mathrm{m}^{2}$ obese, were used in classification of nutrition status. CD4 cell counts were reported as the number of cells in a cubic millimeter of blood. The WHO immunity grading system on bases of CD4 cell counts was used to classify the level of immunosuppression of study participants (table 1).

The prevalence of wasting was calculated as the proportion of those with BMI less than $18.5 \mathrm{~kg} / \mathrm{m}^{2}$, and its relationship with gender and immunity was evaluated. The relation of nutrition status (wasting) with gender and immunity (CD4 count) was also examined. Differences in the proportion of wasting between men and women, and at different CD4 levels were tested with the chi-square tests. Correlation between CD4 cell count and nutrition status was obtained using Pearson's correlation coefficient. Receiver Operating Characteristic (ROC) curves were constructed to assess the relationship of nutrition status with CD4 count (immunity level), and to determine the cutoff point which would predict immune deficiency with optimal sensitivity and specificity.

Table 1. WHO Classification of CD4 level in relation to immune suppression.

\begin{tabular}{ll}
\hline Severity of immune suppression & CD4 count \\
\hline No significant immune suppression & $>500 / \mathrm{mm} 3$ \\
Mild immune suppression & $350-499 / \mathrm{mm} 3$ \\
Advanced immune suppression & $200-349 / \mathrm{mm} 3$ \\
Severe Immune suppression & $<200 / \mathrm{mm} 3$ \\
\hline
\end{tabular}

\subsection{Ethical Approval}

Ethical clearance for this research was obtained from Addis Ababa city administration health bureau ethical clearance board. A letter of permission and support was obtained from the board. Written consent was taken from study participants using forms designed for this study.

\section{Result}

\subsection{Demographic and Anthropometric Characteristics}

A total of 395 HIV infected adults attending pre-ART chronic care clinics were recruited during the study period, July to August, 2014. Only 9 individuals refused involvement in the study. Non-response rate was only 2\%. 229 (58\%) were females and $136(42 \%)$ were males. Most of the study participants (90\%) lived in Addis Ababa. The average duration of follow up in chronic care was 3.5 years. The average age of the study participants was 28 years with men slightly older than women. The mean weight was $56 \mathrm{Kg}$ (53 $\mathrm{kg}$ for women and $64 \mathrm{~kg}$ for men). Mean height was $169 \mathrm{~cm}$ for all study participants: $166 \mathrm{~cm}$ and $173 \mathrm{~cm}$ for women and men respectively. The mean body mass index (BMI in $\mathrm{kg} / \mathrm{m}^{2}$ ) was $19.6 \pm 1.8$ : female $19.2 \pm 1.5$ and male $19.9 \pm 1.7$ (table 2).

\subsection{Nutritional Status}

In this study, the prevalence of wasting (BMI $<18.5 \mathrm{~kg} / \mathrm{m}^{2}$ ) was $27.1 \%$. Two third of the study participants $(67.8 \%)$ had normal nutrition status (BMI 18.5-24.49 kg/m²) (Table 3). The prevalence of wasting varies by gender significantly $(\mathrm{p}=0.00)$. One third of women in the program had BMI below the lowest acceptable value of WHO cutoff point for normal nutrition status $\left(\mathrm{BMI}=18.5 \mathrm{~kg} / \mathrm{m}^{2}\right)$. Less proportion of men $(18.1 \%)$ were wasted compared to women $(33.6 \%)$. There was significant difference in proportion of normal nutrition status by gender $(\mathrm{p}=0.00)$. More proportion of men $(77.1 \%)$ had normal nutrition status than women (61.1\%). 5.1\% of study participants were either overweight or obese with majority of 
them being in the overweight category. Unlike wasting, by gender $(\mathrm{p}=0.16)$. overweight/obesity $\left(\mathrm{BMI} \geq 25 \mathrm{~kg} / \mathrm{m}^{2}\right)$ did not significantly vary

Table 2. Demographic profile of the study population, Addis Ababa, 2014.

\begin{tabular}{llll}
\hline Variable & Overall $(\mathbf{n}=\mathbf{3 9 5})(\boldsymbol{m e a n} \pm$ SD) & Females $(\mathbf{n}=\mathbf{2 2 9})(\mathbf{m e a n} \pm$ SD) & Males $(\mathbf{n}=\mathbf{1 6 6})(\mathbf{m e a n} \pm \mathbf{S D})$ \\
\hline Age $($ years $)$ & $28 \pm 4$ & $30 \pm 3$ & $25 \pm 2$ \\
Weight $(\mathrm{kgs})$ & $56 \pm 5.9$ & $53 \pm 5.7$ & $60 \pm 6.2$ \\
Height $(\mathrm{cms})$ & $169 \pm 20.7$ & $166 \pm 18.8$ & $173 \pm 22.9$ \\
Body mass index $(\mathrm{kg} / \mathrm{m} 2)$ & $19.6 \pm 1.8$ & $19.2 \pm 1.5$ & $19.9 \pm 1.7$ \\
Follow up duration (years) & $2.5 \pm 1.1$ & $2.2 \pm 1.3$ & $2.8 \pm 0.8$ \\
\hline
\end{tabular}

Table 3. Gender wise prevalence of malnutrition among HIV infected adults, Addis Ababa, 2014.

\begin{tabular}{lllll}
\hline Nutrition Status & Total number of study participants N (\%) & Women N (\%) & Men N (\%) & P value \\
\hline Study population & 395 & 229 & 166 & \\
Wasting & $107(27.1 \%)$ & $77(33.6 \%)$ & $30(18.1 \%)$ & 0.00 \\
Normal & $268(67.8 \%)$ & $140(61.1)$ & $128(77.1 \%)$ & 0.00 \\
Overweight/obese & $20(5.1 \%)$ & $12(5.2 \%)$ & $8(4.8 \%)$ & 0.16 \\
\hline
\end{tabular}

\subsection{Clinical Characteristics}

The majority of the study participants were on WHO clinical stage 2. The mean CD4 count was 386 cells $/ \mathrm{mm}^{3}$. Most study participants (77.2\%) had only mild immune suppression with CD4 cell count in the range 350-499 cells $/ \mathrm{mm} 3$. The proportion of women with mild immune suppression $(57.2 \%)$ is significantly lower than the corresponding value in men $(66.2 \%, p=0.04)$. Likewise, the proportion of women with normal immunity was lower as compared to men $(\mathrm{p}=0.02)$. Advanced immunosuppression was more associated with women. $29.7 \%$ of women in the pre-ART program had CD4 cell count below 350 cells $/ \mathrm{mm}^{3}$, which according to WHO classification corresponds to advanced immune suppression. In men, the proportion of advanced immune suppression (12.4\%) was less than half the figure in women (29.7\%). Thus, advanced immune suppression was significantly more prevalent in women than men $(\mathrm{p}=0.00)$. Only $0.8 \%$ of study participants had severe immune suppression (CD4 count less than $200 \mathrm{cel} 1 / \mathrm{mm}^{3}$ ).

Table 4. Gender wise level of immunosuppression, Addis Ababa, 20143.4 Immunity and Nutrition Status.

\begin{tabular}{lllll}
\hline CD4 count & Overall $(\mathbf{n}=\mathbf{3 9 5})$ mean \pm SD (\%) & Females $(\mathbf{n}=\mathbf{2 2 9})$ mean \pm SD(\%) & Males $(\mathbf{n}=\mathbf{1 6 6})$ mean \pm SD(\%) & P value \\
\hline$<200 \mathrm{cell} / \mathrm{mm} 3$ & $3(0.8 \%)$ & $2(0.9 \%)$ & $1(0.6 \%)$ & 0.09 \\
$200-349 \mathrm{cell} / \mathrm{mm} 3$ & $89(22.5 \%)$ & $68(29.7 \%)$ & $21(12.7 \%)$ & 0.00 \\
$350-499 \mathrm{cell} / \mathrm{mm} 3$ & $241(61.0 \%)$ & $131(57.2 \%)$ & $110(66.3 \%)$ & 0.04 \\
$\geq 500 \mathrm{cell} / \mathrm{mm} 3$ & $62(15.7 \%)$ & $28(12.2 \%)$ & $34(20.5 \%)$ & 0.02 \\
\hline
\end{tabular}

We examined the relationship between CD4 cell count and nutritional status. Table 5 shows nutrition status at different levels of immunity. Higher rates of advanced to severe immune suppression were observed among individuals with wasting $(\mathrm{P}<0.001)$ compared to those with normal and above normal nutrition status. There was a moderate correlation between wasting and CD4 cell count $(\mathrm{r}=0.4, \mathrm{P}<0.005)$. The sensitivity and specificity of wasting to predict severe and/or advanced immune suppression was $62 \%$ and $64 \%$ respectively. The sensitivity and specificity of wasting to predict mild immune suppression was $56 \%$ and $59 \%$ respectively. Furthermore, the area under the ROC Curve for wasting and advanced immune suppression was 0.68 (95\% CI 0.61-0.75), and for wasting and mild immune suppression 0.61 (95\% CI $0.57-0.65)$.

\section{Discussion}

The impact of HIV infection on nutrition was identified early in the epidemic with wasting being one of the most visible signs of malnutrition in patients who progress to acquired immunodeficiency syndrome (AIDS). Many studies indicated that malnutrition including underweight and wasting constituted major health problems among HIV infected individuals $(2,3,5)$. The overall prevalence of wasting in this population of HIV-infected, antiretroviral naive adults was $27.1 \%$ which was cause for concern. Our findings were similar to rates of under nutrition among HIV-infected adults reported from other parts of Ethiopia and Africa. The prevalence of wasting, defined as body mass index (BMI) $<18.5 \mathrm{~kg} / \mathrm{m}^{2}$, in adults with HIV infection in Sub-Saharan Africa was in the range $20-40 \%(15,16,17)$. However, the findings of this study were lower than those reported among HIV infected individuals in Asia, which was in the range 60 to $68 \%$ for wasting. Studies from developed and developing countries consistently show that malnutrition, as assessed by low BMI, is a strong independent predictor of mortality among people living with HIV $(13,18)$. A study in Tanzania showed the risk of mortality increased as BMI decreased. One year mortality was $13.7 \%, 21.0 \%$ and $46.8 \%$ in patients with normal, mild-moderate, severe malnutrition respectively. One year estimated risk of mortality was 3.42 and 1.5 times higher 
than normal in severe and moderate malnourished individuals respectively $(16,17,18,19)$.

Table 5. Nutrition status versus CD4 cell count, Addis Ababa, 2014.

\begin{tabular}{lllll}
\hline \multirow{2}{*}{ Nutrition status } & \multicolumn{2}{l}{ Level of immune suppression } & & CD4 350-499 \\
\cline { 2 - 5 } & CD4<200 & CD4 200-349 & CD4 $\geq \mathbf{5 0 0}$ \\
\hline $\mathrm{BMI}<18.5 \mathrm{~kg} / \mathrm{m}^{2}$ & 2 & 62 & 31 & 12 \\
$\mathrm{BMI} 18.5-24.49 \mathrm{~kg} / \mathrm{m}^{2}$ & 1 & 24 & 197 & 46 \\
$\mathrm{BMI} \geq 25 \mathrm{~kg} / \mathrm{m}^{2}$ & 0 & 4 & 12 & 4 \\
\hline
\end{tabular}

Poor nutritional status in PLWH is associated with disease progression, increased morbidity, and reduced survival even when antiretroviral therapy is available $(11,12)$. HIV and malnutrition have a cumulative effect in damaging the immune system and worsening nutritional status. People infected with HIV are more vulnerable to malnutrition than the general population $(2,5)$. The impaired nutritional status of patients with HIV is partially caused by the reduction in calorie intake, the occurrence of opportunistic diseases and the hypercatabolic action of the body in an attempt to control viral replication and recompose the immune system $(5,6)$. The rise in metabolic rate due to HIV infection is $10 \%$ and $20-30 \%$ higher in asymptomatic and symptomatic adults respectively. This increase in energy expenditure will often lead to weight loss. Thus, wasting may occur despite individuals maintaining their usual food intake after acquiring HIV and during all stages of the disease. Besides, through reduction of food intake, impairment of digestion, malabsorption and food insecurity, HIV progressively impairs nutrition status $(6,7,8)$.

Nutrition care is regarded as an essential component of the

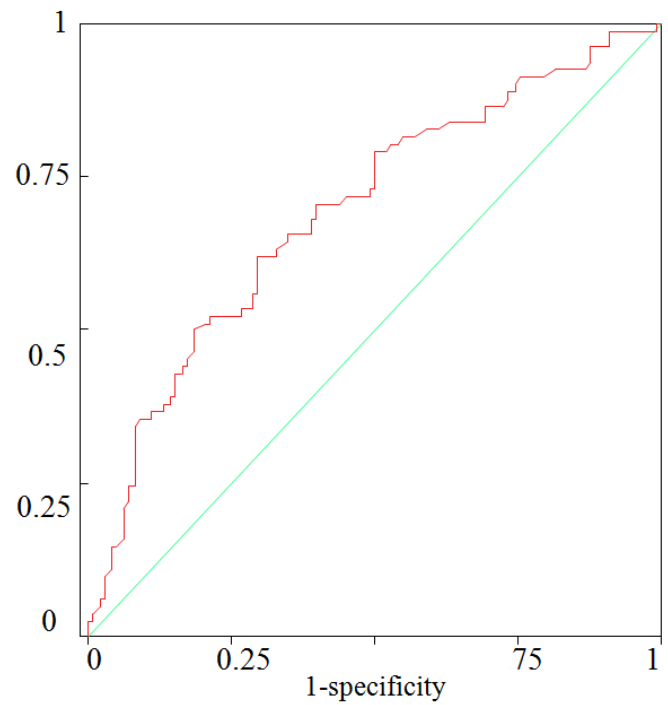

comprehensive response to HIV/AIDS and recommendations have been made to integrate nutrition assessment, counseling and support (NACS) with existing HIV service packages (22). Routine nutrition screening can facilitate prompt treatment and dietary interventions which in turn can reduce the frequency and duration of opportunistic infections as well as prevent weight loss. Evidences showed that unintentional weight loss can progress even when nutrition interventions are provided (24). However, nutrition interventions early in the disease course or before the development of opportunistic infections can prevent unintentional weight loss successfully (27).

Wasting significantly varied by gender with more proportion of women malnourished than men. Almost a third of the women $(33.6 \%)$ were wasted while the figure in men was only $12 \%$. The increased risk of wasting in women may be due to combination of factors like low socioeconomic status, health care access and other issues related with gender inequality (30).

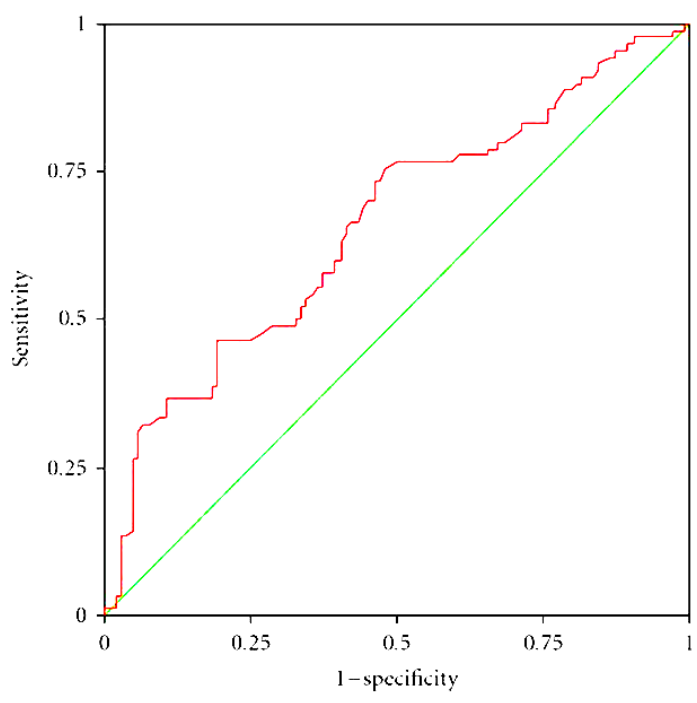

Figure 1. ROC curve between (a) wasting and advanced immune suppression, and (b) wasting and mild immune suppression.

In our study, we observed that immune suppression was significantly associated with wasting. Wasting was associated with lower mean CD4 cell count, indicating more advanced disease was associated with being wasted. By the time they reach a stage of advanced immunodeficiency, approximately three-quarters were wasted. The CD4 counts were lower in those with wasting compared to the other groups (not wasted). The proportion of individuals with normal nutrition decreased with lowering of CD4 cell count. This was because of the fact that malnutrition aggravates the effect of HIV on individuals by further weakening the immune system and increasing vulnerability to opportunistic infections, thereby accelerating the course of the HIV infection $(11,12)$. Malnutrition is usually encountered at the advanced phase of HIV infection. Anthropometric measurements like BMI are lower in symptomatic HIV patients as classified by WHO HIV disease stages, and PLWH with more than $350 \mathrm{CD} 4$ cells/mm3 were more well-nourished than those with less than or equal to 350 


\section{CD4 cells/mm3 (22).}

Despite immune status and wasting showed a fair correlation $(\mathrm{r}=0.4, \mathrm{p}=0.00)$, the presence of wasting alone could not be used to predict disease severity very accurately. Among those with wasting, though the majority had CD4 cell count below 350, almost one fourth of study participants in the group had CD4 $>350$. The sensitivity and specificity of wasting alone in predicting immune status was not very satisfactory. The area under the ROC curve for both mild and advanced immune suppression was in the range of 0.59-0.68, indicating poor diagnostic accuracy. Because malnutrition is common at all stages of HIV disease $(6,7,8)$, wasting cannot be used as a surrogate marker for predicting disease stage or severity of HIV associated immunosuppression. Hence, there is a need to cautiously use wasting in clinical staging of HIV infected adults as its sensitivity and specificity in predicting immunodeficiency are not satisfactory.

\section{Conclusion and Recommendations}

In summary, we have found that there was high prevalence of wasting among HIV-infected antiretroviral naïve adults in Ethiopia. Given the fact that under nutrition aggravates the course of HIV infection, the prevailing high level of malnutrition showed by this study was of significant concern. Routine nutrition screening can facilitate prompt treatment and dietary interventions, which in turn can reduce the frequency and duration of opportunistic infections as well as prevent weight loss. Nutrition interventions early in the disease course or before the development of opportunistic infections can prevent unintentional weight loss successfully and reduce morbidity and mortality. Hence, the existing efforts of ministry of health to avail nutrition assessment, counselling and support (NACS) services should be strengthened. There was moderate correlation between wasting and immune suppression. However, the sensitivity and specificity of wasting alone in prediction of HIV associated immune suppression was not strong enough to consider wasting as an independent surrogate marker of CD4 cell level. Wasting may occur even at relatively higher CD4 levels.

\section{Acknowledgements}

The authors are grateful to the staff of the HIV/AIDS division of Zewditu hospital for their assistance during data collection. The authors would also like to express our gratitude to participants of this study.

\section{Reference}

[1] Koethe JR, Heimburger DC. Nutritional aspects of HIV-associated wasting in sub-Saharan Africa. Am J Clin Nutr 2010, 91:1138S-1142S.

[2] Peter Katona, Judit Katona. The Interaction between Nutrition and Infection. Clin Infect Dis. 2008, 46:1582-1588.
[3] Burgin J, Nichols S, Dalrymple N. The nutritional status of clinical attendees living with HIV/AIDS in St. Vicente and Grenadines. West Indian Med J 2008, 57:438-443.

[4] Ludy MJ, Hendricks K, Houser R, Chetchotisad P, Anunnatsiri S. Body composition in adults infected with human immunodeficiency vírus in Khon Kaen, Thailand. Am J Trop Med Hyg 2005, 73:815-819.

[5] L. Salomon J, Truchis P, Melchior JC. Nutrition and HIV infection. Br J Nutr 2002, 87: 111-119.

[6] Melchior JC, Salmon D, Rigaud D, Leport C, Bouvet E, Detruchis $\mathrm{P}$, et al. Resting energy expenditure is increased in stable, malnourished HIV infected patients. Am J Clin Nutr 1991, 53(2):437-41.

[7] Melchior JC, Raguin G, Boulier A, Bouvet E, Rigaud D, Matheron $\mathrm{S}$, et al. Resting energy expenditure in human immunodeficiency virus-infected patients: comparison between patients with and without secondary infections. Am J Clin Nutr 1993, 57(5):614-9.

[8] Dannhauser A, van Staden AM, van der Ryst E, Nel M, Marais $\mathrm{N}$, Erasmus E, et al. Nutritional status of HIV-1 seropositive patients in the Free State Province of South Africa: anthropometric and dietary profile. Eur J Clin Nutr 1999, 53:165-73.

[9] Palermo T, Rawat R, Weiser SD, Kadiyala S. 10. Food Access and Diet Quality Are Associated with Quality of Life Outcomes among HIV-Infected Individuals in Uganda. PLoS One. 2013, 8(4):e62353.

[10] Food Assistance Programming in context of HIV. FANTA Project, Academy for Educational Development, Washington, DC; 2007.

[11] Scrimshaw NS, SanGiovanni JP. Synergism of nutrition, infection, and immunity: an overview. Am J Clin Nutr 1997, 66(2):464S-477S12.

[12] Mangili A, Murman DH, Zampini AM, Wanke CA. Nutrition and HIV infection: review of weight loss and wasting in the era of highly active antiretroviral therapy. Clin Infect Dis 2006; 42(6):836-42

[13] A Bussmann H, Wester CW, Ndwapi N, et al. Five-year outcomes of initial patients treated in Botswana's National Antiretroviral Treatment Program. AIDS 2008, 22:2303-11.

[14] Tariku M, Kemal Z. Prevalence of malnutrition in HIV infected indioviduals in St. Peter Hospital, Addis Ababa, Ethiopia. Hindai Publishing 2013, 12: 145-151.

[15] Dannhauser A, van Staden AM, van der Ryst E, Nel M, Marais N, Erasmus E, et al. Nutritional status of HIV-1 seropositive patients in the Free State Province of South Africa: anthropometric and dietary profile. Eur J Clin Nutr 1999, 53:165-73.

[16] Zachariah R, Fitzgerald M, Massaquoi M, Pasulani O, Arnould L, Makombe S, et al. Risk factors for high early mortality in patients on antiretroviral treatment in a rural district of Malawi. AIDS 2006, 20:2355-60.

[17] Stringer JS, Zulu I, Levy J, et al. Rapid scale-up of antiretroviral therapy at primary care sites in Zambia: feasibility and early outcomes. JAMA 2006, 296:782-93. 
[18] Zachariah R, Fitzgerald M, Massaquoi M, et al. Risk factors for high early mortality in patients on antiretroviral treatment in a rural district of Malawi. AIDS 2006, 20:2355-60.

[19] Johannessen A, Naman E, Ngowi BJ, et al. Predictors of mortality in HIV-infected patients starting antiretroviral therapy in a rural hospital in Tanzania. BMC Infect Dis 2008, 8:52-60.

[20] Kotler DP. Wasting syndrome: nutritional support in HIV infection. AIDS Res Hum Retroviruses 1994, 10:931-934.

[21] World Health Organization. Conference on Nutrition and HIV/AIDS in Africa: Evidence, lessons and recommendations for action. Executive summary of a scientific review. Geneva: WHO, 2005.

[22] Somarriba G, Neri D, Schaefer N, Miller LT. The effect of aging, nutrition and exercise during HIV infection. HIV/AIDS. Research and Palliative Care. 2010, 2:191-20.

[23] Zachariah R, Reid SD, Chaillet P, Massaquoi M, Schouten EJ, Harries AD. Viewpoint: why do we need a point-of-care CD4 test for low-income countries? Trop Med Int Health. 2011, $16: 37-41$

[24] Colebunders R, Mann JM, Francis H, Bila K, Izaley L, Kakonde N, et al. Evaluation of a clinical case-definition of acquired immunodeficiency syndrome in Africa. Lancet. 1987, $1: 492-4$.

[25] De Cock KM, Colebunders R, Francis H, Nzilambi N, Laga M, Ryder RW, et al. Evaluation of the WHO clinical case definition for AIDS in rural Zaire. AIDS. 1988, 2:219-21.

[26] Malamba SS, Morgan D, Clayton T, Mayanja B, Okongo M, Whitworth J. The prognostic value of the World Health Organiazation staging system for HIV infection and disease in rural Uganda. AIDS. 1999, 13:255-62.

[27] FANTA publications, Ethiopia Nutrition and HIV Tools: Food and nutrition. The federal democratic of Ethiopia, Ministry of Health National Nutrition and HIV/AIDS Implementation Reference Manual. 2008

[28] FHAPCO. Monthly HIV Care and ART Update: Update as of end of February, 2015. Addis Ababa, Ethiopia web site: www.HAPCO.GOV.et.

[29] Child health and nutrition in Ethiopia. Analysis of EDHS 2011, Addis Ababa, Ethiopia, 2013.

[30] Central Statistical Agency and ICF International. Ethiopia Demographic and Health Survey. Central Statistical Agency, Addis Ababa, Ethiopia; ICF International, Calverton, Md, USA, 2012 